\title{
recildunds
}

Revista Cientifica Mundo de la Investigación y el Conocimiento

\author{
Martha Isabel Arcos Gutiérrez a ; Jimmy Andrés Barros Segovia ${ }^{\text {b }}$; Christian David \\ Cevallos Mendoza ${ }^{c}$; Rossibel Berenisse Ollague Armijos ${ }^{\text {d }}$
}

Tratamiento conservador en fractura de clavícula

Conservative treatment in clavicle fracture

Revista Científica Mundo de la Investigación y el Conocimiento. Vol. 3 núm. 4., diciembre, ISSN: 2588-073X, 2019, pp. 571-585

DOI: $10.26820 /$ recimundo/3.(4).diciembre.2019.571-585

URL: http://recimundo.com/index.php/es/article/view/682

Código UNESCO: 3205 Medicina Interna

Tipo de Investigación: Artículo de Revisión

(C) RECIMUNDO; Editorial Saberes del Conocimiento, 2019

Recibido: 15/09/2019

Aceptado: 23/11/2019

Publicado: 30/12/2019

Correspondencia: marthica12@msn.com

a. Médico; Investigador Independiente; Guayaquil, Ecuador; marthica12@msn.com

b. Médico; Investigador Independiente; Guayaquil, Ecuador; jbarros@ contigo.com.ec

c. Médico; Investigador Independiente; Guayaquil, Ecuador; drchrisdace@ gmail.com

d. Médico; Investigador Independiente; Guayaquil, Ecuador; dra_ollague@ hotmail.com 


\section{Tratamiento conservador en fractura de clavícula}

Vol. 3, núm. 4., (2019)

Martha Isabel Arcos Gutiérrez; Jimmy Andrés Barros Segovia; Christian David Cevallos Mendoza; Rossibel Berenisse Ollague Armijos

\section{RESUMEN}

Las fracturas de clavícula tradicionalmente han sido tratadas de forma conservadora, estas suponen entre 2.6 y $4 \%$ de todas las fracturas del adulto y $35 \%$ de las lesiones de la cintura escapular. Entre un 70-80\% de estas fracturas corresponden al tercio medio de clavícula. Su adecuado diagnóstico y tratamiento son fundamentales ya que este hueso contribuye a la fuerza y estabilidad del brazo, actuando como punto de origen e inserción muscular y a su vez, ofreciendo protección a estructuras neurovasculares importantes, entre otras funciones. El objetivo fundamental de este estudio es plasmar lo relativo al tratamiento conservador en las fracturas de clavícula. El diseño de investigación que se llevó a cabo es de tipo documental o bibliográfico. El tratamiento de estas fracturas va a depender, en gran medida, de su clasificación. Históricamente, el manejo conservador o no quirúrgico en las fracturas de clavícula es el más usado, quizás motivado a que la mayoría de estas ocurren en el tramo distal de este hueso, es decir, son de tipo I y presentan características que pueden ser sanadas con el uso único de inmovilización. No obstante, existen casos, en donde ciertas condiciones de la fractura la hacen candidata a un manejo quirúrgico. El manejo conservador de la fractura de clavícula se realiza básicamente por medio de la inmovilización de esta área mediante un cabestrillo (con férula o tablilla, según sea el caso), tratamiento medicamentoso de la inflamación y el dolor, y fisioterapia para recuperar la fuerza y la movilidad del hombro. Este tratamiento a lo largo del tiempo ha generado muy buenos pronósticos y excelente evolución.

Palabras Claves: Tratamiento; Conservador; Clasificación; Fractura; Clavícula. 


\title{
Tratamiento conservador en fractura de clavícula
}

Vol. 3, núm. 4., (2019)

Martha Isabel Arcos Gutiérrez; Jimmy Andrés Barros Segovia; Christian David Cevallos

Mendoza; Rossibel Berenisse Ollague Armijos

\begin{abstract}
Clavicle fractures have traditionally been treated conservatively, these represent between 2.6 and $4 \%$ of all adult fractures and $35 \%$ of scapular waist injuries. Between $70-80 \%$ of these fractures correspond to the middle third of the clavicle. Its proper diagnosis and treatment are fundamental since this bone contributes to the strength and stability of the arm, acting as a point of origin and muscular insertion and in turn, offering protection to important neurovascular structures, among other functions. The main objective of this study is to capture the conservative treatment of clavicle fractures. The research design that was carried out is documentary or bibliographic. The treatment of these fractures will depend, to a large extent, on their classification. Historically, conservative or non-surgical management of clavicle fractures is the most used, perhaps due to the fact that most of these occur in the distal section of this bone, that is, they are type I and have characteristics that can be healed with the unique use of immobilization. However, there are cases, where certain fracture conditions make it a candidate for surgical management. Conservative management of the clavicle fracture is basically done by immobilization of this area by means of a sling (with splint or splint, as the case may be), medicated treatment of inflammation and pain, and physiotherapy to regain strength and Shoulder mobility This treatment over time has generated very good prognoses and excellent evolution.
\end{abstract}

Keywords: Treatment; Conservative; Classification; Fracture; Clavicle. 


\section{Tratamiento conservador en fractura de clavícula}

Vol. 3, núm. 4., (2019)

Martha Isabel Arcos Gutiérrez; Jimmy Andrés Barros Segovia; Christian David Cevallos Mendoza; Rossibel Berenisse Ollague Armijos

\section{Introducción.}

El uso de terapias clásicas con una eficacia reconocida y la evasión de tratamientos agresivos, han sido el estándar de oro a lo largo de los años para el tratamiento de innumerables patologías, las cuales se encuentran íntimamente relacionadas con una menor gravedad del cuadro y, por ende, un mejor pronóstico.

Tal es el caso de las fracturas de clavícula, las cuales tradicionalmente, en la mayoría de los casos y, sobre todo en pacientes pediátricos, tienen un buen pronóstico y excelente evolución cuando son tratadas de manera conservadora.

La fractura de clavícula es una lesión frecuente, generalmente en niños y adultos jóvenes. La clavícula es un hueso en forma de S itálica que conecta la parte superior del esternón con el omóplato. Entre las causas más frecuentes de la fractura de clavícula se encuentran: caídas, traumatismos por accidentes de tránsito y lesiones deportivas. Otra causa es el proceso de nacimiento, donde algunos bebés nacen con una clavícula fracturada. Alguno de los síntomas que la definen son:

- Dolor que aumenta al mover el hombro

- Hinchazón

- Sensibilidad

- Hematomas

- Protuberancia en el hombro o cerca de este 


\section{Tratamiento conservador en fractura de clavícula}

Vol. 3, núm. 4., (2019)

Martha Isabel Arcos Gutiérrez; Jimmy Andrés Barros Segovia; Christian David Cevallos Mendoza; Rossibel Berenisse Ollague Armijos

- Un chasquido o chirrido cuando se intenta mover el hombro

- Rigidez o incapacidad para mover el hombro

- Con frecuencia, los niños recién nacidos no moverán el brazo por varios días después de una fractura de clavícula relacionada con el parto. (Mayo Clinic, 2018)

Para Lenza, Buchbinder, Johnston, Ferrari, \& Faloppa (2019) "la clavícula actúa como un puente a través del frente del tórax que conecta el brazo y la caja torácica". Tiene la función de coadyuvar en la estabilización del hombro y a su vez permite el movimiento libre del brazo, es punto de adherencia de varios músculos, y parte del aparato musculoesquelético que usa el cuerpo humano en la respiración. También cumple la función de proteger los nervios y vasos sanguíneos de esa zona, y tiene una función estética importante para la apariencia física de la persona. El sitio más común de la fractura de clavícula es el tercio medio de este hueso y por lo general, suele ocurrir en jóvenes y adultos mayores. En la mayoría de los casos es causada por una caída directa sobre el lado exterior del hombro. (p. 7)

Con respecto a las estadísticas, González, Lopez, García, \& Marco (2013) reportan en su trabajo que las fracturas de clavícula suponen entre 2.6 y $4 \%$ de todas las fracturas del adulto y $35 \%$ de las lesiones de la cintura escapular. Entre un 70-80\% de estas fracturas corresponden al tercio medio de clavícula. Con respecto a la edad media de los pacientes con fractura de clavícula es de 33 años y $70 \%$ de ellos son varones. Las fracturas de tercio medio de la clavícula tradicionalmente se han tratado de forma conservadora, incluso aquellas que presentan un desplazamiento importante. (p. 289) 


\section{Tratamiento conservador en fractura de clavícula}

Vol. 3, núm. 4., (2019)

Martha Isabel Arcos Gutiérrez; Jimmy Andrés Barros Segovia; Christian David Cevallos Mendoza; Rossibel Berenisse Ollague Armijos

De igual manera, Ferrás, Lastra, \& Chacón (2017) destacan entre las funciones de la clavícula su contribución a la fuerza y estabilidad del brazo, actuando como punto de origen e inserción muscular y a su vez, ofreciendo protección a estructuras neurovasculares importantes. "El ascenso de la porción lateral de la clavícula aumenta la tracción sobre la primera costilla, a través del ligamento costoclavicular y el músculo subclavio, conduciendo al desplazamiento del tórax en sentido cefálico, que corresponde a una inspiración”. (p. 264)

Khan LA, 2009 citado por el Instituto Mexicano del Seguro Social (2012) afirma que las complicaciones que se presentan frecuentemente posterior a una fractura de clavícula son alteraciones vasculares, pulmonares y neurológicas, así como retardo de consolidación, seudoartrosis, perdida de tejidos blandos, consolidación viciosa, rigidez articular y re fractura. (p. 8)

Las fracturas de clavícula pueden ser tratadas de manera conservadora o con reparación quirúrgica. En tal sentido, la decisión acerca del mejor enfoque para su manejo, debe ser tomada entre el paciente y el cirujano, siendo importante la discusión de cuestiones pertinentes tales como: la situación clínica (grado de desplazamiento de la fractura y otras características), las necesidades del paciente (tipo del trabajo actividad de ocio), y las preferencias del paciente (tras la evaluación de los riesgos y beneficios de la cirugía versus el tratamiento conservador). (Banco de Preguntas Preevid, 2013)

La presente investigación tiene como objetivo compilar y plasmarla de forma sencilla, información relacionada con el manejo conservador de la fractura de clavícula.

\section{Materiales y Métodos.}




\section{Tratamiento conservador en fractura de clavícula}

Vol. 3, núm. 4., (2019)

Martha Isabel Arcos Gutiérrez; Jimmy Andrés Barros Segovia; Christian David Cevallos Mendoza; Rossibel Berenisse Ollague Armijos

El presente estudio se limitó a la búsqueda y revisión sistemática de material bibliográfico digitalizado, el cual se compiló y plasmó en una síntesis relacionada con el tratamiento conservador de la fractura de clavícula, en razón de lo que dicha investigación es de tipo documental bibliográfica.

Las principales bases de datos relacionadas con las áreas médicas que fueron usadas para la búsqueda fueron: MedlinePlus, Mayo Clinic, SciELO, ELSEVIER, entre otras.

Los descriptores utilizados para la búsqueda fueron los siguientes: "Fractura de clavícula", "clavícula", "clasificación de las fracturas de clavícula", "clavícula" y "tratamiento conservador de las fracturas de clavícula". Los resultados fueron filtrados según los criterios de idioma español, relevancia, correlación temática y una fecha de publicación de los últimos ocho años, sin descartar el tipo de material bibliográfico.

\section{Resultados.}

\section{Clasificación de las fracturas}

Generalmente, en fracturas de clavícula en niños y adolescentes, el tratamiento dependerá del tipo de fractura sufrido y de su gravedad. En su mayoría, estas fracturas pueden ser tratadas con simples medidas de confort hasta su curación.

Para Carvajal, Gómez, Borja, \& Sepúlveda (2016) la meta primaria del tratamiento de la fractura de clavícula consiste en la restauración de "la función previa del hombro comprometido. Existe completo acuerdo en la literatura en que la indicación para manejo con reducción cerrada es la presencia de fractura no desplazada y no complicada"; no obstante, no existe acuerdo 


\section{Tratamiento conservador en fractura de clavícula}

Vol. 3, núm. 4., (2019)

Martha Isabel Arcos Gutiérrez; Jimmy Andrés Barros Segovia; Christian David Cevallos Mendoza; Rossibel Berenisse Ollague Armijos

general en cuanto a indicaciones para manejar fracturas con reducción abierta y fijación interna. (p. 92)

Existen varias clasificaciones descritas para estas fracturas. El sistema de clasificación más ampliamente aceptado, es el de Allman (1967), el cual divide las fracturas de clavícula en base a su localización sobre el hueso, no obstante, a pesar de ser la clasificación más usada, esta brinda poca información con respecto a la elección de tratamiento según la fractura y, por ende, el resultado terapéutico que pueda resultar.

Las fracturas claviculares, según Allman, pueden ser:

- Grupo I Fractura del tercio medio

- Grupo II Fractura del tercio distal

- Grupo III Fractura del tercio medial. (Ferrás, Lastra, \& Chacón, 2017, p. 264)

Jiménez et al. (2014) amplía la información de esta clasificación al describirlas de la siguiente manera:

La fractura tipo I o fractura diafisaria, que se trata ortopédicamente, salvo que existan condicionantes quirúrgicos como un acortamiento superior o igual a $20 \mathrm{~mm}$, fractura abierta o patológica con parálisis del músculo trapecio o fractura irreductible, afectación cutánea, neurovascular, disociación escapulotorácica o una gran separación entre los fragmentos; otras indicaciones son el hombro flotante, deportistas o 


\section{Tratamiento conservador en fractura de clavícula}

Vol. 3, núm. 4., (2019)

Martha Isabel Arcos Gutiérrez; Jimmy Andrés Barros Segovia; Christian David Cevallos Mendoza; Rossibel Berenisse Ollague Armijos

personas con grandes requerimientos funcionales. La fractura tipo II afecta al tercio lateral y se han descrito hasta cinco subtipos. El tratamiento quirúrgico se indica por el riesgo de pseudoartrosis. Finalmente, las de tipo III afectan al tercio medial y suelen tratarse ortopédicamente, salvo que existan desplazamientos posteriores. (p. 30)

Por otra parte, Ávila et al. (2015) describe la clasificación de Neer, quien define las fracturas distales de clavícula como aquellas localizadas lateral esa la inserción más medial en clavícula del ligamento trapezoide y las subdivide en 3 tipos:

- El tipo I son fracturas no desplazadas, con los ligamentos CC intactos, que estabilizan el fragmento medial. Son fracturas estables con buen pronóstico.

- El tipo II son fracturas desplazadas en las que el fragmento medial asciende y aumenta el riesgo de seudoartrosis. Suelen requerir tratamiento quirúrgico. Este tipo II se divide, a su vez, en 2 subtipos:

- IIA cuando la fractura es medial a ambos ligamentos $\mathrm{CC}$

- IIB cuando la fractura es lateral, pero asocia rotura del ligamento conoide.

- El tipo III son fracturas intraarticulares con afectación de la articulación AC, atravesándola o asociando luxación. Estos casos pueden ser quirúrgicos también. De hecho, si el fragmento distal es pequeño y genera dolor se puede practicar su exéresis quirúrgica abierta o artroscópica, con resultados favorables. (p. 50) 


\section{Tratamiento conservador en fractura de clavícula}

Vol. 3, núm. 4., (2019)

Martha Isabel Arcos Gutiérrez; Jimmy Andrés Barros Segovia; Christian David Cevallos Mendoza; Rossibel Berenisse Ollague Armijos

\section{Tratamiento Conservador}

El manejo conservador o no quirúrgico de la fractura de clavícula consistente en la aplicación de vendaje o cabestrillo con la finalidad de inmovilizar esta zona durante el período de tiempo necesario para que la fractura logre la consolidación.

Un cabestrillo es un dispositivo que se usa para apoyar y mantener quieta (inmovilizada) una parte del cuerpo que se encuentre lesionada. Estos pueden ser utilizados en muchas lesiones diferentes, no obstante, se usan con mayor frecuencia en casos de fractura o una dislocación de un brazo o un hombro. La finalidad de una tablilla o férula previa al cabestrillo, consiste en evitar el movimiento del hueso roto o dislocado. En casos de lesión que requiera ser entablillada, primero se coloca la férula y luego el cabestrillo. "Las férulas reducen el dolor y ayudan a prevenir un mayor daño a los músculos, los nervios y los vasos sanguíneos. Las férulas o tablillas también reducen el riesgo de que se abra una herida cerrada". (Enciclopedia Médica ADAM, 2017)

En casos de fracturas de clavícula no desplazadas, la opción de manejo optima consiste comúnmente en la inmovilización con cabestrillo o un brace en forma de 8 , aplicado en la fase aguda, la cual proporciona una inmovilización que debe permanecer entre dos a seis semanas, de acuerdo con el nivel de confort del paciente. En pacientes adultos esta inmovilización puede extenderse hasta por tres meses. En este caso, el retorno a los deportes o a la actividad pesada es permitida entre la semana cuatro a seis, posterior a la consolidación clínica o radiológica. Un trabajo ligero que implique actividad con el brazo por encima del nivel de la cabeza debe ser iniciado una vez que el confort lo permita, generalmente, esto sucede de dos a cuatro semanas 


\section{Tratamiento conservador en fractura de clavícula}

Vol. 3, núm. 4., (2019)

Martha Isabel Arcos Gutiérrez; Jimmy Andrés Barros Segovia; Christian David Cevallos Mendoza; Rossibel Berenisse Ollague Armijos

después de la consolidación. “Algunos estudios han mostrado altas tasas de no consolidación sintomática $(51,4 \%)$ y función del hombro reducida por el test de Constant $(48,6 \%)$, después del tratamiento no quirúrgico". Asimismo, es importante mencionar que, para las fracturas tratadas de forma conservadora, resulta inevitable algún grado de deformidad y acortamiento. (Carvajal, Gómez, Borja, \& Sepúlveda, 2016, p. 92)

El Instituto Mexicano del Seguro Social (2012) acerca del tratamiento conservador de fracturas de clavícula menciona en su guía de práctica clínica que:

Históricamente la mayoría de las fracturas de clavícula en principio han recibido tratamiento conservador. No se recomienda en ningún momento, realizar maniobras de reducción a nivel de clavícula, porque las fracturas de clavícula usualmente son inestables y no hay forma de brindar soporte externo. Ningún vendaje reduce una fractura desplazada. El vendaje tipo Velpau y el vendaje en"8" pueden producir lesión dérmica y/o compresión del paquete neurovascular. Se recomienda el uso de "cabestrillo" con la finalidad de inmovilizar la extremidad del lado en que se encuentra la fractura de la clavícula, lo que permitirá su consolidación. El tiempo de recuperación de las fracturas de clavícula que son manejadas en forma conservadora es en promedio de 90 días. En los adultos mayores, la consolidación de las fracturas de clavícula es más lenta; aumenta el tiempo de consolidación aproximadamente un 33\%, debido a los cambios metabólicos propios de la edad, en estos pacientes la consolidación de la fractura tarda en promedio 120 días Se 


\section{Tratamiento conservador en fractura de clavícula}

Vol. 3, núm. 4., (2019)

Martha Isabel Arcos Gutiérrez; Jimmy Andrés Barros Segovia; Christian David Cevallos Mendoza; Rossibel Berenisse Ollague Armijos

recomienda realizar control radiológico a las 6 y 12 semanas, para

evaluar la presencia y grado de consolidación, una vez observado el callo

óseo, el paciente puede regresar a las actividades diarias, solo con

restricción en actividades de carga y tracción. (p. 5)

En pediatría, aquellas fracturas más sencillas, donde los fragmentos óseos permanecen alineados, el tratamiento consiste en seguir las siguientes recomendaciones:

- Hielo. Para ayudar a controlar el dolor y la inflamación asociados a una fractura de clavícula, aplique bolsas de hielo sobre el área afectada durante los dos o tres días posteriores a la lesión. De todos modos, no aplique el hielo directamente sobre la piel, ya que podría lesionarla.

- Sostén del brazo. Es importante mantener el brazo inmovilizado mediante un cabestrillo. Esto ayudará a controlar el dolor y a mantener al hueso en su sitio durante el proceso de curación.

- Medicación. Es importante que la medicación la prescriba el pediatra tratante (ibuprofeno, paracetamol) a fin de aliviarle el dolor.

- Fisioterapia. Mientras el brazo permanece inmovilizado, lo más probable es que pierda fuerza muscular y que la gama de movimientos del hombro afectado se reduzca. Cuando los huesos se le empiecen a curar, se debe comenzar a realizar ejercicios de movimientos suaves a fin de reducir el agarrotamiento mientras se lleva puesto el cabestrillo. Cuando el hueso se haya curado por completo, es posible que el pediatra 


\section{Tratamiento conservador en fractura de clavícula}

Vol. 3, núm. 4., (2019)

Martha Isabel Arcos Gutiérrez; Jimmy Andrés Barros Segovia; Christian David Cevallos Mendoza; Rossibel Berenisse Ollague Armijos

recomiende un programa más intenso de ejercicios de rehabilitación para recuperar toda la fuerza y flexibilidad del hombro afectado. (Atanda, 2014)

Igualmente, "la clavícula de un recién nacido quebrada durante el parto usualmente sana solo controlando el dolor y manipulando con cuidado al bebé”. (Mayo Clinic, 2018)

\section{Conclusión.}

El manejo de las fracturas de clavícula debe ser individual para cada paciente, en razón de las características de la fractura, así como de las expectativas del paciente. Es importante para la toma de decisión terapéutica tener en cuenta aspectos tales como: características individuales del paciente y su cuadro clínico, preferencias del paciente y evaluación de la relación riesgos beneficios, de cada una de las opciones.

El tratamiento conservador o no quirúrgico es el manejo más usado a lo largo de la historia en las fracturas de clavícula, quizás motivado a que la mayoría de estas ocurren en el tramo distal de este hueso, es decir, son de tipo I y presentan características que pueden ser sanadas con el uso único de inmovilización. No obstante, existen casos, en donde ciertas condiciones de la fractura la hacen candidata a un manejo quirúrgico.

El manejo conservador de la fractura de clavícula se realiza básicamente por medio de la inmovilización de esta área mediante un cabestrillo (con férula o tablilla, según sea el caso), tratamiento medicamentoso de la inflamación y el dolor y fisioterapia para recuperar la fuerza y la movilidad del hombro. Este tratamiento históricamente ha generado muy buenos pronósticos y rara vez, alguna complicación. 


\section{Tratamiento conservador en fractura de clavícula}

Vol. 3, núm. 4., (2019)

Martha Isabel Arcos Gutiérrez; Jimmy Andrés Barros Segovia; Christian David Cevallos

Mendoza; Rossibel Berenisse Ollague Armijos

\section{Bibliografía.}

Atanda, A. (Septiembre de 2014). Fractura de clavícula. (KidsHealth, Editor) Recuperado el 02 de Diciembre de 2019, de https://www.brennerchildrens.org/KidsHealth/Parents/ParaPadres/Seguridad-y-primeros-auxilios/Fractura-de-clavicula.htm

Ávila, J. L., Moros, S., Edo, O., García, C., García, C., \& del Olmo, T. (2015). Fracturas de clavícula distal. Artroscopia y Cirugía Articular, 22(1), 49-53. Recuperado el 29 de diciembre de 2019, de https://www.researchgate.net/publication/282534004_Fracturas_de_clavicula_distal

Banco de Preguntas Preevid. (2013). Indicaciones del tratamiento quirúrgico en pacientes adultos con fracturas cerradas del tercio medio de la clavícula. Recuperado el 01 de Enero de 2020, de https://www.murciasalud.es/preevid/19714

Carvajal, M., Gómez, C., Borja, W., \& Sepúlveda, L. E. (2016). FRACTURAS DIAFISIARIAS DE LA CLAVÍCULA: REVISIÓN DE LA EVIDENCIA PUBLICADA. Biosalud, 15(1), 87-97. Recuperado el 15 de Diciembre de 2019, de http://www.scielo.org.co/pdf/biosa/v15n1/v15n1a10.pdf

Enciclopedia Médica ADAM. (16 de Octubre de 2017). Cómo hacer un cabestrillo. Recuperado el 21 de Diciembre de 2019, de https://medlineplus.gov/spanish/ency/article/000017.htm

Ferrás, E., Lastra, A., \& Chacón, M. (2017). Fractura bilateral de clavícula. Revista Científica Villa Clara, 21(3), 263-267. Recuperado el 20 de Diciembre de 2019, de http://scielo.sld.cu/pdf/mdc/v21n3/mdc15317.pdf

González, A. M., Lopez, Y., García, C., \& Marco, F. (2013). Fracturas de tercio medio de clavícula. Resultados tras osteosíntesis. Ata Ortopédica Mexicana, 27(5), 288-292. $\begin{array}{llllll}\text { Recuperado el } 20 \text { de } & \text { Diciembre }\end{array}$ https://www.medigraphic.com/pdfs/ortope/or-2013/or135b.pdf

IMSS. (2012). Tratamiento de la Fractura de Clavícula en el Adulto: Guía de Referencia Rápida. Dirección de Prestaciones Médicas, Unidad de Atención Médica. México: Instituto Mexicano del Seguro Social. Recuperado el 30 de Diciembre de 2019, de http://www.imss.gob.mx/sites/all/statics/guiasclinicas/584GRR.pdf

Instituto Mexicano del Seguro Social . (2012). Tratamiento de Fracturas de Clavícula en el Adulto: Evidencias y Recomendaciones. Dirección de Prestaciones Médicas, Unidad de Atención Médica. División de Excelencia Clínica. Recuperado el 20 de Diciembre de 2019, de http://www.imss.gob.mx/sites/all/statics/guiasclinicas/584GER.pdf

Jiménez, A., Santos, F., Zurera, M., Najarro, F., Chaqués, F., \& Pérez, S. (2014). Fracturas claviculares tratadas con placas. Trauma Fund. MAPFRE, 25(1), 29-36. Recuperado el 20 


\section{Tratamiento conservador en fractura de clavícula}

Vol. 3, núm. 4., (2019)

Martha Isabel Arcos Gutiérrez; Jimmy Andrés Barros Segovia; Christian David Cevallos Mendoza; Rossibel Berenisse Ollague Armijos

de Diciembre de 2019, de https://www.mapfre.com/fundacion/html/revistas/trauma/v25n1/docs/articulo5.pdf

Lenza, M., Buchbinder, R., Johnston, R., Ferrari, B., \& Faloppa, F. (22 de Enero de 2019). Intervenciones quirúrgicas versus conservadoras para el tratamiento de las fracturas de clavícula en adolescentes y adultos. Cochrane Database of Systematic Reviews . $\begin{array}{llllll}\text { Recuperado el } 10 \text { de } & \text { Diciembre }\end{array}$ https://www.cochrane.org/es/CD009363/intervenciones-quirurgicas-versusconservadoras-para-el-tratamiento-de-las-fracturas-de-clavicula-en

Mayo Clinic. (27 de Septiembre de 2018). Fractura de clavícula. Recuperado el 15 de Diciembre de 2019, de https://www.mayoclinic.org/es-es/diseases-conditions/brokencollarbone/symptoms-causes/syc-20370311

$$
\text { (c) } \frac{1}{\mathrm{BY} \text { NC }(2)}
$$

RECONOCIMIENTO-NOCOMERCIAL-COMPARTIRIGUAL

CC BY-NC-SA

ESTA LICENCIA PERMITE A OTROS ENTREMEZCLAR, AJUSTAR Y CONSTRUIR A PARTIR DE SU OBRA CON FINES NO COMERCIALES, SIEMPRE Y CUANDO LE RECONOZCAN LA AUTORÍA Y SUS NUEVAS CREACIONES ESTÉN BAJO UNA LICENCIA CON LOS MISMOS TÉRMINOS. 